\section{Cahiers de Narratologie}

Analyse et théorie narratives

15 | 2008

Récits et genres historiques

\title{
Du drame à la tragédie : l'évolution de la conception de l'histoire chez Augustin Thierry
}

\section{Paule Petitier}

\section{(2) OpenEdition}

1 Journals

Édition électronique

URL : http://journals.openedition.org/narratologie/819

DOI : 10.4000/narratologie.819

ISSN : 1765-307X

Éditeur

LIRCES

\section{Référence électronique}

Paule Petitier, « Du drame à la tragédie : l'évolution de la conception de l'histoire chez Augustin Thierry », Cahiers de Narratologie [En ligne], 15 | 2008, mis en ligne le 15 décembre 2008, consulté le 14 novembre 2019. URL : http://journals.openedition.org/narratologie/819; DOI : 10.4000/narratologie. 819

Ce document a été généré automatiquement le 14 novembre 2019.

\section{c) (†) $९$}

Cahiers de Narratologie - Analyse et théorie narratives est mis à disposition selon les termes de la licence Creative Commons Attribution - Pas d'Utilisation Commerciale - Pas de Modification 4.0 International. 


\title{
Du drame à la tragédie : l'évolution de la conception de l'histoire chez Augustin Thierry
}

\author{
Paule Petitier
}

1 «Plus elle fit d'instances et de prières pour que Merowig fût accueilli avec hospitalité et pût vivre en paix auprès d'elle, plus ceux qui gouvernaient au nom du jeune roi se montrèrent durs et intraitables. Ils avaient pour prétexte le danger d'une rupture avec le roi de Neustrie; ils ne manquèrent pas de s'en prévaloir, et leur condescendance pour les affections de la reine se borna à congédier simplement le fils d'Hilperik, sans lui faire violence ou le livrer à son père. $»^{1}$

2 Bien qu'il ait lutté pour restituer leur graphie germanique aux noms des Francs conquérants de la Gaule, hérissant de consonnes barbares les trop familiers « Clovis » et "Mérovée », Augustin Thierry écrit dans une langue classique et élégante, sobre mais non dépourvue de noblesse. N'usant pratiquement pas de la métaphore, économe en qualificatifs, encore plus en phrases exclamatives ou interrogatives, il ne recule pas devant les parallélismes, les antithèses, les cosntructions qui soulignent les rapports logiques et préfère la tournure abstraite. Bref, son style est assez intellectuel bien que l'on ait fait de lui le maître de l'école pittoresque en histoire².

3 Dans les Récits des temps mérovingiens, cette langue très classique contraste particulièrement avec l'objet auquel elle s'applique : la barbarie des conquérants de la Gaule. On ne tombe pas dans le burlesque car les personnages qui sont au centre du récit sont des rois, des reines, des hommes de pouvoir, mais il existe un décalage entre la noblesse de la narration d'une part et de l'autre la crudité et la bassesse des motifs, des passions, voire des actions.

4 « Fredegonde nourrissait contre les enfants de son mari une haine de belle-mère, qui, à défaut de tout autre exemple, aurait pu devenir proverbiale. Tout ce que leur père avait pour eux de tendresse et de complaisance excitait sa jalousie et son dépit. Elle désirait leur mort, et celle de Theodebert, tué l'année précédente, lui avait causé une grande joie. » (p. 99) 
5 Autre contraste : celui d'un projet historique s'appliquant à une période de confusion historique voire d'absence d'histoire. Augustin Thierry, en choisissant de traiter du VI siècle, tenait une espèce de gageure, l'époque étant considérée comme peu intéressante par sa barbarie même. Période de transition, de décomposition entre l'Empire romain et la naissance de la France, elle ne présentait à l'historien que le triste tableau d'une décadence. Au mieux pouvait-on y observer l'hybridation de la civilisation romaine, de l'énergie gauloise et de la barbarie franque. Mais ce n'était pas un moment où les lignes générales de l'histoire étaient clairement perceptibles, du moins pour un historien comme Thierry. Comment saisir une orientation, un dessein général dans « le déchaînement des passions individuelles »? C'est pour cela même que Thierry disait avoir choisi la forme narrative : «je ne puis employer un autre moyen pour retracer un temps comme celui-là où l'histoire n'a aucun caractère de généralité et se disperse dans les faits privés » («Introduction»).

Le contraste entre narration et diégèse

6 Le récit donne à voir une époque de désorganisation. À la mort de Chlother, ses quatre fils, Gonthramm, Hilperick et Sighebert se sont partagé ses terres, mais sans qu'intervienne bien sûr l'idée d'un espace national. Un découpage bizarre a produit de multiples enclaves, les villes, indépendamment de leur site, ont été réparties entre les quatre frères. La possession de ces villes est à l'origine dans le troisième récit de conflits et de combats, les villes d'Aquitaine, héritage du roi d'Austrasie étant convoitées par le roi de Neustrie Hilpérik. À cette confusion territoriale s'ajoute l'absence totale de coordination entre ceux qui détiennent le pouvoir. Le fils du roi n'en fait qu'à sa tête : chargé d'une mission militaire, il s'arrête à Tours, rançonne un allié de son père, et part à Rouen accomplir ses projets amoureux. Fredegonde, l'épouse d'Hilperik, ne songe qu'à son propre pouvoir, son plus cher souhait est la mort de son beau-fils Merowig. Elle le poursuit d'une haine indépendante des conflits qui opposent le père et le fils. Les actions de tous les Francs sont soumises à la passion et à l'humeur : si Hilperik ne succombe pas aux charmes de Brunehilde, c'est parce que la passion de l'or est chez lui plus forte que celle de la chair. Mais son fils, lui, tombe immédiatement amoureux de la reine. La terreur peut s'emparer entièrement de l'esprit des Francs, obnubilant elle aussi la raison. Hilperik soupçonne son fils de comploter contre lui et en éprouve « une terreur panique ». Complètement dominés par leurs passions, les Francs ignorent ou transgressent continuellement toute forme de loi. Merowig et Brunehilde n'hésitent pas à se marier malgré les règles canoniques qui interdisent l'union d'un homme avec la veuve de son oncle. Hilperik et Gontramm-Bose violent la parole donnée. Le roi de Neustrie cherche à contourner par l'intimidation ou par des négociations avec Saint Martin le droit d'asile dans les églises. Merowig exige qu'on lui donne les eulogies ${ }^{3}$ alors qu'il a visiblement rompu son vœu religieux; il menace de tuer quelqu'un dans l'église si l'on n'exauce pas sa volonté. Agissant impulsivement sous l'empire d'accès de passions ou d'humeur, les Francs sont par ailleurs incapables de projets à long terme, leur comportement change avec leur humeur. La découverte du trésor de Brunehilde modifie les dispositions d'Hilperik envers sa prisonnière et l'engage à la clémence. Humeurs changeantes, arbitraire des volontés individuelles, rencontres inattendues, absence d'organisation générale impliquant de constantes rébellions, "complication[s] d'événements impossibles à prévoir» (p. 111), voilà la matière ingrate dont le narrateur doit rendre compte. 
7 Or la narration semble justement se caractériser par des qualités exactement opposées aux vices de la barbarie.

Premièrement elle dégage des lois. La conduite des barbares a beau sembler uniquement réglée par l'humeur, la passion ou l'intérêt individuel, cela n'empêche pas la généralisation.

9 "Dans ce déclin de la Gaule vers la barbarie, l'impatience et l'oubli de toute règle étaient la maladie du siècle ; et, pour tous les esprits, même les plus éclairés, la fantaisie individuelle ou l'inspiration du moment tendait à remplacer l'ordre et la loi.» (p. 101-102)

10 Le narrateur formule ici la loi d'une époque paradoxale, où l'oubli de la généralité est la règle générale. Cet énoncé explique le comportement de l'évêque Praetextatus qui accepte de marier, contre les règles canoniques, Merowig et Brunehilde. Il s'agit bien d'une loi, valable pour tous, pour les Gallo-Romains comme pour les Francs, puisqu'elle explique la conduite répréhensible d'un homme d'origine gauloise. Cependant, plus la barbarie est marquée, donc plus les conduites sont apparemment déréglées, plus la règle générale s'applique. Lorsque le narrateur évoque l'Austrasie, le royaume le plus oriental et donc le plus proche des mœurs originelles des Germains, il rappelle le principe énoncé plus haut :

11 "L'absence de tout frein légal et le déchaînement des volontés individuelles s'y faisaient sentir plus fortement que dans aucune autre portion de la Gaule. Il n'y avait à cet égard aucune distinction de race ni d'état; Barbares ou Romains, prélats ou chefs militaires, tous les hommes qui se croyaient forts par le pouvoir ou la richesse luttaient à qui mieux mieux de turbulence et d'ambition. » (p. 129)

12 La narration a le souci de rattacher les actions à des coutumes, de restaurer le rapport du particulier au général. Lors de la dégradation de Merowig, le narrateur expose quelle coutume donne sens à cette punition. Il explique aussi le rituel des eulogies.

13 Alors que les barbares se caractérisent par leur vision à courte portée, la narration ouvre des perspectives temporelles. Lorsque Brunehilde et Merowig se réfugient dans l'église de Saint-Martin pour échapper à la colère d'Hilperik, le narrateur insère une digression suggérant que le style gothique trouverait son origine dans ces modestes constructions en bois. C'est une anticipation de cinq siècles.

14 Deuxièmement, la narration s'oppose aussi au portrait du barbare donné dans le récit par son extrême souci de rationalisation. Il y a même une sorte de paradoxe à ce que les actions des barbares, présentées comme le résultat d'une conduite purement impulsive et irréfléchie, soient à ce point motivées. Par exemple lorsque Merowig accepte la suggestion perfide de son compagnon Gonthramm-Bose de sortir des limites de l'asile religieux, le narrateur fournit une triple explication :

15 «Le besoin d'espace et d'air libre que ressentent si vivement les emprisonnés parlait au cœur de Merowig, et sa facilité de caractère lui faisait approuver sans examen tout ce que proposait son ami. Il accueillit avec la vivacité de son âge cette invitation attrayante. » (p. 122) La narration entrelace constamment les éléments explicatifs à l'exposé des faits.

17 "À cette nouvelle, qui lui enlevait toute espérance de réunir sans guerre à son royaume le royaume de son frère, Hilperik, furieux de voir échouer le projet qui lui était le plus cher, fit 
diligence pour arriver à Paris et s'assurer au moins de la personne et des trésors de Brunehilde.» (p. 97, c'est moi qui souligne)

D'abord, l'exposé des motifs, qui ne craint pas une certaine redondance entre la proposition relative explicative et l'apposition à Hilperik ; puis l'articulation avec le but recherché. Ce souci de motivation conduit Thierry à entrer dans l'intériorité de ses personnages en reconstruisant leurs sentiments et leurs desseins. Cette dérive subjective est cependant contenue par le caractère strictement pragmatique de la psychologie prêtée aux personnages. Il s'agit uniquement d'expliquer des décisions, des actes. Thierry sacrifie d'ailleurs à la prudence que lui commande sa position d'historien en employant à plusieurs reprises l'alternative : « soit pour obtenir en retour des terres $\mathrm{du}$ fisc, soit pour s'assurer une protection dans le désordre qui menaçait leur pays » ( $\mathrm{p}$. 96). Parfois, l'alternative présentant deux causes inverses dévoile le caractère systématique de l'exigence de motivation : «Soit que Brunehilde s'attendît à un pareil témoignage d'affection, soit que l'arrivée du fils d'Hilperik fût pour elle une cause de surprise $[. .] ».($ p. 101). Le principe de la narration est que tout acte a une cause, peut être expliqué, fût-ce par la «fantaisie individuelle».

Troisièmement la rapidité des actions dans la diégèse (destinée à exprimer l'impatience des barbares et le tempo frénétique de ce moment historique) contraste avec la pondération voire la redondance de la narration :

20 "À cette révocation inespérée des ordres qui la retenaient en exil, Brunehilde s'empressa de quitter Rouen et la Neustrie au plus vite, comme si la terre eût tremblé sous ses pieds. Dans la crainte du moindre retard, elle brusqua ses préparatifs de voyage [...]» (p. 105 , c'est moi qui souligne)

21 Enfin, si l'univers barbare se caractérise par la désorganisation et l'instabilité, la narration met fortement en relief son pouvoir de configuration. Elle réunit et organise les faits de façon à produire des effets très nets de convergence. Elle excelle à rendre la fluctuation et la désorganisation par l'évocation de mouvements qui prennent de l'ampleur puis se retournent dans une nouvelle configuration. Ainsi le début du troisième récit évoque-t-il un premier mouvement de bascule. Après le meurtre de Sighebert, une dynamique s'enclenche en faveur d'Hilperik. Thierry rapproche des éléments épars dans la chronique de Grégoire de Tours pour obtenir un effet d'amplification. On voit se recomposer un ensemble de forces autour d'Hilperik :

22 "Hilperik, victorieux par un fratricide, s'avançait vers Paris pour s'emparer de la famille et des trésors de son frère. Non seulement tous les Neustriens revenaient à lui sans exception, mais les principaux des Austrasiens commençaient à être gagnés, et, se rendant sur son passage, ils lui juraient fidélité, soit pour obtenir en retour des terres $\mathrm{du}$ fisc, soit pour s'assurer une protection dans le désordre qui menaçait leur pays. Un seigneur, nommé Godin ou Godewin, reçut, pour prix de sa défection, de grands domaines dans le voisinage de Soissons; et le gardien de l'anneau royal ou du grand sceau d'Austrasie, le référendaire Sig ou Sigoald, donna le même exemple, qui fut suivi par beaucoup d'autres. » (p. 96)

Thierry emploie ensuite la technique du « contre-champ » en revenant à Brunehilde. Le désarroi initial (supposé par le narrateur) et la solitude de la reine ne semblent être là que comme prélude à un redéploiement. Là où Grégoire de Tours indique seulement que Gondobald fit regagner l'Austrasie au jeune héritier, Thierry suppose Brunehilde à l'initiative de cette action. Le récit «prend» alors. Le rappel de l'échec de Gondobald 
"deux années auparavant» accentue la fonction "configurative ", c'est le moment où l'on passe au rassemblement des données. Le récit se reconstitue autour de Brunehilde.

«Convaincue de l'impossibilité de fuir avec sa famille et ses bagages, elle conçut l'idée de sauver au moins son fils qui, tout enfant qu'il était, faisait trop d'ombrage à l'ambition de Hilperik pour que sa vie fût épargnée. L'évasion du jeune Hildebert fut préparée dans le plus grand secret par le seul ami dévoué qui restât à sa mère ; c'était le duc Gondobald, le même qui, deux années auparavant, avait si mal défendu le Poitou contre l'invasion des Neustriens. » (97)

Enfin le paragraphe se termine par l'évocation d'une recomposition des forces dans la réalité historique: "la défection cessa et les Franks orientaux s'empressèrent de relever leur royauté nationale. Il y eut à Metz une grande assemblée des seigneurs et des guerriers de l'Austrasie » (p. 97)

En passant d'un personnage à un autre, le récit traduit l'éclatement de l'action en fonction des individualités, mais paragraphe après paragraphe, la narration fait preuve de sa virtuosité à configurer les faits et les motifs. Le récit est conçu comme une succession de "nœuds narratifs $»^{4}$ régie le plus souvent par le principe du renversement.

Par l'ensemble de ses traits (mise en avant de la loi, inscription de l'individuel dans une généralité, explication rationnelle des actes et des faits, efficacité de son organisation), la narration contraste avec le tableau de la barbarie donné par la diégèse. Cette opposition trouve un écho à l'intérieur même de la diégèse dans les notations qui différencient les Gallo-Romains des Francs. En effet, selon Thierry, au VI siècle, les différences entre les deux "races", celle des conquérants et celle des vaincus, sont encore perceptibles. Les Gallo-Romains gardent l'empreinte de la civilisation. Le général Desiderius, "grand seigneur d'origine gauloise", se distingue de «ses concurrents d'origine barbare " par «quelque largeur dans les vues et d'assez grands talents militaires » (p. 106). Ayant à combattre un autre Gaulois, "le célèbre Eonius Mummolus ", il livre " une bataille rangée, une de ces batailles qu'on ne voyait plus en Gaule depuis que la tactique romaine avait fait place à la guerre d'escarmouche et de partisans, la seule que comprissent les barbares»(p. 107). Cette bataille apparaît d'ailleurs comme l'un des rares faits d'ampleur qui ait "frapp[é] l'imagination des contemporains » (p. 108). Bien qu'ils se soient affrontés, les deux hommes s'estiment et s'unissent « dans une entreprise qui ne tendait à rien moins qu'à fonder un nouveau royaume sur le territoire gaulois » (ibid.). Les rares prolepses du texte sont réservées à ces personnages de « race » gallo-romaine.

«Cinq ans après, de Dax à Poitiers et d'Alby à Limoges, toutes les villes appartenaient au roi de Neustrie; et le Romain auteur de cette conquête, installé dans Toulouse, l'ancienne capitale des Visigoths, exerçait, avec le titre de duc, une sorte de viceroyauté. » (ibid.)

En Austrasie, le Romain Lupus est un "administrateur sévère et vigilant, nourri des vieilles traditions du gouvernement impérial» (p. 130). Certains Gallo-Romains partagent donc les qualités dont fait preuve la narration. Ils sont capables de rigueur, d'organisation, de largeur de vue. L'un d'eux se distingue particulièrement par des 
vertus proches de celles du narrateur, il s'agit de l'évêque Grégoire de Tours. Son portrait en fait une vivante antithèse de l'absence de sens moral des barbares.

"Quelque péril qu'il y eût alors, au milieu de la société bouleversée, à défendre la cause des faibles et des proscrits contre la force brutale et la mauvaise foi des hommes puissants, Grégoire montrait, dans cette lutte sans cesse renouvelée, une constance que rien ne pouvait lasser, et une dignité prudente mais intrépide.» (p. 110)

31 La constance, la dignité, l'intrépidité manquent totalement à la famille de Hilpérik. Une qualité caractérise particulièrement Grégoire, le respect de la légalité. Il veille « avec fermeté au maintien des droits de son église et surtout du droit d'asile» (ibid.). Lors de l'irruption de Merowig dans la basilique de Saint-Martin, il est sensible à la «double infraction aux lois ecclésiastiques» que manifeste l'étrange apparence du jeune homme. En concédant les eulogies à Merowig, il conserve le souci de « sauver au moins les formes légales ». Enfin, après lui avoir accordé l'asile, il se "hât[e] de remplir certaines formalités qu'exigeait la loi romaine » (p. 115). Thierry suit de près dans le récit de cet épisode le texte même de Grégoire dans son Histoire des Francs. On peut constater cependant qu'il souligne beaucoup le souci des formes légales par rapport au texte original :

32 «La messe achevée, le prince se fit connaître, et nous demanda les eulogies. Nous répondîmes d'abord par un refus; mais il s'écria qu'on ne pouvait pas le retrancher ainsi, sans jugement de la communion des fidèles. Après avoir examiné sa demande d'après le droit canonique, du consentement de mon collègue qui m'assistait, je lui donnai les eulogies.

Cet événement causa de grands malheurs à la ville de Tours. J'envoyai mon diacre, accompagné de mon neveu Nicétus, que ses affaires appelaient à Soissons, pour informer le roi Chilpéric de la fuite de Mérovée. $»^{5}$

Les adaptations auxquelles se livre Thierry par rapport à sa source consistent par exemple à censurer les passages dans lesquels s'exprimait une croyance trop naïve de Grégoire dans l'intervention directe des puissances surnaturelles. Thierry reprend dans Grégoire de Tours l'histoire de l'intervention menaçante de Rokolen pour obtenir l'extradition de Gonthramm-Bose. Mais alors que Grégoire attribue à la main de Dieu une jaunisse qui frappe Rokolen au milieu de ses dévastations et un malaise providentiel qui l'atteint au moment où il touche le seuil de la basilique sacrée, Thierry évoque seulement des causes rationnelles : "selon toute apparence, [il] avait trop peu de monde avec lui pour tenter quelque chose de sérieux contre la population d'une grande ville» (p. 111). Alors que Grégoire évoque comme une manifestation naturelle du surnaturel le rêve dans lequel un ange lui annonce l'extinction de la lignée de Chilpéric, Thierry prend soin de rationaliser presque à outrance le fait. Le rêve n'est plus que la traduction en images d'un pressentiment moral. La " révélation de l'avenir » est rattachée au souci du bien public dont fait preuve l'évêque :

«De tristes réflexions le préoccupèrent, au milieu du chant des psaumes [la religion est écartée de la production du rêve (PP)]; et, lorsque, après l'office des vigiles, voulant prendre un peu de repos, il se fut mis au lit dans un appartement voisin de l'église, les crimes dont cette église semblait devoir être le théâtre dans la guerre contre nature allumée entre le père et le fils, tous les malheurs qu'il prévoyait sans pouvoir les conjurer, le poursuivirent en quelque sorte jusqu'au moment où il s'endormit. Durant 
le sommeil, les mêmes idées, traduites en images terribles, se présentèrent encore à son esprit. » (p. 118)

Le travail d'adaptation auquel se livre Thierry contribue donc à rendre Grégoire de Tours plus proche du narrateur, dont il finit par être la mise en abyme. Par ce procédé, Thierry justifie sa confiance dans sa source principale: Grégoire apparaît comme un narrateur fiable puisqu'il partage les qualités et les valeurs du narrateur moderne. La relation du narrateur à l'objet de sa narration reproduit celle de Grégoire vis-à-vis de la conduite des Francs. Mais surtout Grégoire devient le représentant de l'histoire dans une époque sans histoire. Même lorsque l'histoire semble au point mort, il subsiste une conscience historique qui élabore le récit des faits de son point de vue. À défaut de se faire, l'histoire peut encore s'écrire. Elle est cependant réduite à la conscience, vidée de substance. Il n'y a plus d'opposition dramatique, mais une dissociation entre l'intellect (identifié à l'histoire) et la barbarie des Mérovingiens.

Effacement du drame

37 Le mot « drame » fait partie du vocabulaire romantique et si l'on peut dire de la vision du monde romantique. Il apparaît comme une notion structurant l'interprétation de la réalité aussi bien dans l'histoire que dans le roman, voire dans la philosophie. Chez Thierry, il renvoie à une conception de l'histoire qui, sous la Restauration, faisait du conflit un élément moteur. On retrouve constamment le mot chez Michelet pour désigner tant la nature des conflits historiques que leur sens. Pour Balzac le «drame » est la clef du dynamisme romanesque.

Le drame lie fortement le destin des personnages à leur environnement matériel. Or sans les Récits des temps mérovingiens la narration de Thierry aboutit plutôt à dissocier le destin des personnages de leur environnement matériel. Bien que les changements de lieux soient extrêmement fréquents, Thierry ne se soucie pas de peindre les paysages ou les villes des royaumes francs. On imagine bien le parti qu'un romancier comme Balzac aurait pu tirer de ce "district sauvage qui s'étendait au nord d'Arras vers les côtes de l'Océan » (p. 134), lieu où est tendu le guet-apens fatal à Merowig. Il n'y a qu'à penser à l'utilisation qu'il fait de la topographie de Fougères et du paysage breton dans Les Chouans. Mais Thierry se contente de cette brève notation. Il ne s'attarde guère non plus à décrire le cadre de vie des personnages. S'il donne des précisions sur l'église de bois où se réfugient Brunehilde et Merowig, c'est pour éclairer l'histoire de l'architecture. La prolepse dissocie le lieu et le destin des personnages au lieu de les rattacher comme c'est le cas dans le roman.

L'attention portée au costume est elle aussi symptomatique de l'utilisation nondramatique d'éléments semblant se prêter à un traitement dramatique. Au moment où Merowig fait l'objet d'une première sanction, la perte de sa chevelure et l'ordination, Thierry explique ce châtiment en se référant aux mœurs des Francs. On apprend au passage que le costume du clergé n'est autre que l'habit romain, la différence des états (militaire et religieux) recouvre donc celle des « races» (conquérants et vaincus). Le récit retrouve le motif du costume lorsque Merowig, s'évadant sur le chemin qui devait le mener à sa prison monacale, reprend "le costume tout militaire de sa nation". Thierry énumère alors toutes les pièces de cet habillement. Merowig dissimule le contraste étrange de sa "tête de clerc sur les épaules d'un soldat » en s'enveloppant d'un capuchon, dont une note précise alors l'origine gauloise. Le déguisement de Merowig pourrait être l'amorce d'un épisode romanesque. Mais Thierry ne s'en sert que pour justifier le refus des eulogies opposé à Merowig: la silhouette encapuchonnée 
suscite la défiance des diacres qui distribuent le pain bénit ; ils n'en proposent pas au jeune homme. Furieux, celui-ci se dirige alors vers l'autel et fait un esclandre. «À ces mots, il rejeta en arrière le capuchon de son manteau, et découvrit aux regards des assistants son visage rouge de colère, et l'étrange figure d'un soldat tonsuré.» (p. 114)

41 Au premier degré, le capuchon permet ici un effet dramatique. L'épisode n'est pas dépourvu d'une certaine théâtralité, la tension monte au maximum, jusqu'à l'affrontement entre Merowig et l'évêque. Mais la logique de l'évitement l'emporte sur celle du conflit au moment même de l'acmé dramatique (l'évêque "fit donner à Merowig les eulogies qu'il réclamait»). L'incident illustre encore le clivage entre intellect et instinct (le premier reconnaissant en somme qu'il n'a pas à se mesurer à l'autre). Le vêtement a servi à mettre en évidence une «figure » choquante. Ce terme indique assez que l'intellect dominait déjà au moment même de la théâtralisation. Le vêtement ne particularise pas un individu, il rend visible quelque chose d'assez abstrait, une sorte d'impossibilia, d'atteinte à la logique. La phrase suivante décrypte l'impossibilia en termes de règles: «Le jeune fugitif paraissait devant lui chargé d'une double infraction aux lois ecclésiastiques [...] 》 (ibid.)

On comparera avec une scène analogue de la Conquête de l'Angleterre ( $1^{\mathrm{e}}$ édition en 1825), où le vêtement sert vraiment à dramatiser l'action :

$43 \mathrm{Au} \mathrm{XII}{ }^{\mathrm{e}}$ siècle, le chancelier Guillaume de Longchamp a reçu du roi Richard, parti en croisade, la régence du royaume. Mais son autorité est contestée par les grands et il doit s'enfuir en cachette :

44 «Il sortit de la ville à pied et déguisé, ayant par-dessus ses habits d'homme une jupe de femme et une cape à larges manches, la tête couverte d'un voile d'étoffe épaisse, tenant sous le bras un ballot de toile, et à la main une aune. Dans cet attirail qui était celui des marchandes anglaises de l'époque, le chancelier se rendit vers la mer, et fut obligé d'attendre quelque temps le navire où il devait s'embarquer.

Il s'assit tranquillement sur une pierre avec son ballot sur les genoux; des femmes de pêcheurs qui passaient l'abordèrent en lui demandant le prix de sa toile; mais, faute de savoir un seul mot d'anglais, le chancelier ne répondit rien, ce qui étonna fort les acheteuses. Elles s'éloignèrent cependant; mais d'autres femmes survinrent, aperçurent la toile, et, l'ayant touchée pour l'examiner, firent la même demande que les premières. La prétendue marchande continua de garder le silence, et les femmes renouvelèrent leurs questions : enfin, poussé à bout, le chancelier se mit à rire tout haut, croyant sortir d'embarras par cette espèce de réponse. À ce rire hors de propos, les femmes crurent qu'elles avaient devant elles une personne idiote ou aliénée, et, soulevant son voile pour la reconnaître, découvrirent un visage d'homme fraîchement rasé. Leurs cris de surprise ameutèrent les ouvriers du port ; ceux-ci, joyeux de trouver un objet de risée, se jetèrent sur le personnage déguisé, le tirant par ses habits, le faisant tomber par terre, et s'amusant de ses vains efforts pour leur échapper ou leur faire comprendre qui il était. Après l'avoir traîné quelque temps à travers les cailloux et la boue, les pêcheurs et les matelots finirent par l'enfermer dans une cave, d'où il ne sortit qu'en faisant connaître sa mésaventure aux agents de l'autorité normande. ${ }^{6}$

Dans cette scène, les contradictions du déguisement engendrent une action. Et puis, c'est au peuple saxon que le grand seigneur est confronté, alors que dans l'épisode des Récits, Merowig s'affronte à l'évêque. Ce détail est significatif de la transposition d'un 
conflit en un jugement. La mésaventure du chancelier donne lieu à une action concrète et grotesque ; l'apparition de la tête de Merowig a l'effet, toute proportion gardée, de celle de la Méduse - elle fige la situation.

Une construction dramatique joue sur la convergence de plusieurs intrigues vers un nœud dramatique. Dans Le Père Goriot, les intrigues nouées autour des différents personnages confluent: le duel organisé par Vautrin a lieu le matin même où celui-ci est arrêté, le bal de la vicomtesse de Beauséant coïncide avec la maladie du père Goriot, les malheurs conjugaux des deux sœurs, la décision de la vicomtesse de se retirer du monde. Or, dans les Récits des temps mérovingiens, on n'aboutit pas à cet effet d'unité dramatique. La mise en intrigue est contrariée par un principe narratif presque systématique qui consiste à faire se succéder des actions interrompues, contrecarrées. Une action survient pour en suspendre une autre. Par exemple, Merowig est mandaté par son père pour conquérir les villes d'Aquitaine liées à l'Austrasie. L'amour de Merowig pour Brunehilde lui fait abandonner cette mission. Il se rend à Caen pour épouser la veuve de son oncle. Aussitôt, Hilperik décide de séparer les deux époux en partant lui-même pour Caen. Ou encore : Merowig est châtié et condamné à finir ses jours dans un monastère. Mais cette action est interrompue par l'intervention de Gaïlen qui délivre Merowig. Merowig arrivant à Tours, interrompt la cérémonie de la messe.

La relation entre les différentes actions se fait sur le mode de l'interférence et de l'interruption. Au moment où l'histoire de Gonthramm-Bose se mêle à celle de Merowig, on pourrait penser que se construit un intérêt dramatique. Mais, bien que Thierry annonce une « complication d'événements » (p. 111), l'action reste linéaire.

Enfin il me semble que le drame présuppose une intersubjectivité entre les personnages, y compris ceux qui sont en conflit. Dans Le Père Goriot, Rastignac est capable de partager les émotions de la vicomtesse de Beauséant, de comprendre la détresse du Père Goriot, et même les malheurs conjugaux de Delphine de Nucingen. Dans l'épopée, l'homogénéité axiologique rend inutile d'une certaine façon l'intersubjectivité. Dans le drame, la diversité des valeurs implique une sorte de terrain de rencontre commun dans l'intersubjectivité. Dans le troisième Récit de Thierry, malgré la part importante faite par le narrateur à la reconstitution psychologique, il n'existe effectivement pas d'intersubjectivité. La psychologie enferme chaque personnage dans son monde. Le découpage du récit en paragraphes correspond fréquemment, on l'a noté, à un changement de personnage. Le début du paragraphe présente les espérances, les craintes ou les projets de celui-ci :

50 « À cette nouvelle, qui lui enlevait toute espérance de réunir sans guerre à son royaume le royaume de son frère, Hilperik [...]» (p. 97)

51 «Hilperik, tenace dans ses projets plutôt par lenteur d'esprit que par énergie de caractère, $[. .$.$] songea [. .] ».($ p. 99)

52 "Cette direction n'était pas celle que le jeune homme aurait suivie de préférence s'il eût été libre de marcher à sa fantaisie [...] » (p. 100) 

d'Aquitaine [...]» (p. 102) de son imagination.

La tragédie l'histoire :
«Hilperik se trouvait à Paris, plein d'espérance pour le succès de l'expédition

Les motivations des personnages de barbares, conformément au caractère qui leur est prêté, les ramènent toujours à eux-mêmes. Ce sont d'ailleurs des êtres qui se laissent obnubiler par une idée - Merowig par son amour, Hilperik par la crainte de son fils. Bien que leur psychologie soit très pragmatique et tournée vers l'action, leur égocentrisme les enferme dans la sphère de leurs représentations. La reconstitution de la psychologie des personnages n'est pas placée sous le signe de l'empathie, mais de la déduction. Même la fin de Merowig semble cohérente avec cet enfermement de chacun dans son monde intérieur, faute d'un monde partagé. Son suicide est lié à la terreur née

"Cette imagination d'homme du Nord, triste et rêveuse, qui formait le trait le plus saillant de son caractère, s'exalta peu à peu jusqu'au délire; il fut obsédé par des pensées de mort violente et d'horribles images de tortures et de supplices. Une profonde terreur du sort qui lui était réservé s'empara de lui avec de telles angoisses, que, désespérant de tout, il ne vit de recours que dans le suicide. » (p. 134-135)

6 Il semble bien qu'au moment où Thierry écrit ses Récits, il ait presque abandonné le modèle du drame au profit d'un autre. Après la mort de Sighebert, racontée dans le deuxième des Récits des temps mérovingiens, la voix de l'auteur commente ainsi le sens de

«Telle fut la fin de ce long drame qui s'ouvre par un meurtre et qui se dénoue par un meurtre; véritable tragédie où rien ne manque, ni les passions, ni les caractères, ni cette sombre fatalité qui était l'âme de la tragédie antique, et qui donne aux accidents de la vie réelle tout le grandiose de la poésie. Le sceau d'une destinée irrésistible n'est, dans aucune histoire, plus fortement empreint que dans celle des rois de la dynastie mérovingienne. Ces fils de conquérants à demi sauvages, nés avec les idées de leurs pères au milieu des jouissances du luxe et des tentations du pouvoir, n'avaient dans leurs passions et leurs désirs ni règle ni mesure. Vainement des hommes plus éclairés qu'eux sur les affaires de ce monde et sur la conduite de la vie élevaient la voix pour leur conseiller la modération et la prudence, ils n'écoutaient rien; ils se perdaient faute de comprendre ; et l'on disait : Le doigt de Dieu est là. C'était la formule chrétienne ; mais, à les voir suivre en aveugles, et comme des barques emmenées à la dérive, le courant de leurs instincts brutaux et de leurs passions désordonnées, on pouvait, sans être un prophète, deviner et prédire la fin qui les attendait presque tous. » (p. 94)

Les Mérovingiens sont des espèces d'Atrides ; il s'exterminent en famille. Leur histoire reprend les principes de l'action tragique :

L'aveuglement : les personnages perdent toute lucidité du fait de leurs passions, ce qui les empêche d'ailleurs de voir les passions des autres.

« Hilperik ne s'aperçut de rien [l'amour de Merowig pour Brunehilde], tout occupé qu'il était à faire compter et inventorier les sacs d'or et d'argent [...]» (p. 98)

L'enfermement : outre l'enfermement psychologique dont nous avons parlé, il y a aussi le cercle que dessine autour de certains personnages (de Merowig en particulier) l'asile ecclésiastique. L'espace n'existe presque que comme piège. 

elle s'écrit. Sous la Monarchie de Juillet, l'histoire peut-elle encore être vue comme drame ? Le modèle qui s'était constitué sous la Restauration au moment du conflit entre les deux blocs politiques, libéral et ultra, perdait de son sens une fois le parti libéral au pouvoir, après la Révolution de 1830. Et même, lorsque la frange la plus conservatrice de ce libéralisme eut immobilisé le régime, il devint d'autant plus nécessaire aux historiens en accord avec celui-ci d'atténuer la conception de l'histoire comme conflit. La parution des Récits en 1840 explique en partie la dérive du « drame » vers la tragédie. D'une part, 1840, moment de crise politique, correspond à la démoralisation d'une partie de l'opinion (libéraux démocrates et républicains), au sentiment d'une " panne " de l'histoire. L'absence de substance historique dans les Récits de Thierry peut correspondre à ce désarroi. D'autre part, Thierry reste parfaitement dans l'orthodoxie orléaniste en utilisant pour rendre intelligible le passé le clivage entre instinct et intellect (différenciant les barbares conquérants de l'élite des vaincus gallo-romains), clivage sur lequel s'appuient alors les théoriciens de la classe dominante pour justifier leur domination sur un peuple présenté comme primaire. Les Mérovingiens barbares de Thierry métaphorisent aussi bien les vices de l'aristocratie d'Ancien Régime (dont ils seraient les ancêtres) que le désastre d'un pouvoir exercé par la barbarie populaire.

Le succès officiel des Récits s'explique sans doute par cette orthodoxie. Ils remportent en 1840 le prix Gobert, un prix de 9000 francs destiné à récompenser « le morceau le plus éloquent sur l'histoire de France ». Michelet y était aussi candidat, mais fut repoussé, selon Thierry, « avec une défaveur marquée ».

Trois ans après le succès des Récits de Thierry, le public se détourna du drame romantique en donnant à la Lucrèce de François Ponsard le retentissement qu'il avait refusé la même année aux Burgraves de Victor Hugo. Le retour à la tragédie était bien dans l'air du temps quand on représentait l'histoire. Cette pièce traite d'un sujet politique, le renversement d'un régime despotique et le passage de la monarchie à la république - une république qui éloigne soigneusement la démocratie directe ${ }^{7}$. Or rien ne se joue sur la scène publique, tout est centré sur le foyer. C'est dans la sphère privée que le changement politique va trouver son origine, dans la peinture de la vertu domestique et conjugale de Lucrèce, filant tard le soir bien que patricienne et refusant de se divertir quand son mari est à l'armée. La pièce n'est pas sans rapport avec Lorenzaccio, il s'y trouve aussi un personnage qui feint la dégradation pour couvrir ses desseins politiques, Brute. Mais Brute devient un héros. L'antagonisme politique est rabattu par la fable centrale sur une opposition morale. Sextus, le fils de Tarquin, convoite la vertueuse Lucrèce, il est aussi amoral, dissolu et cruel que les barbares de 
Thierry. Il néglige les livres sacrés que la Sibylle lui propose. Celle-ci en brûle deux et offre le troisième à Brute. Le viol de Lucrèce, mais surtout le terrible châtiment que lui impose son surmoi (tout le monde lui dit qu'elle n'a rien à se reprocher, mais elle se condamne elle-même), déclenche la rébellion contre Tarquin. Dans les Récits de Thierry comme dans Lucrèce, la politique se voit réduite au privé ; Thierry semble le déplorer, mais d'une certaine façon dans les deux cas, histoire et politique sont restreintes à la conscience morale. La pièce de Ponsard se termine juste au moment où les Romains partent pour renverser Tarquin. Il n'y a pas de représentation du combat. L'action centrale reste le suicide de Lucrèce, qui a lieu sur scène (indice d'un vestige de drame ?) mais contribue à faire du "drame » une affaire morale, personnelle et non collective. Une fois la grandeur morale de Lucrèce prouvée par cet acte « sublime " par lequel elle se punit d'une souillure dont elle n'est pas responsable, l'histoire n'a plus besoin d'être représentée.

Outre qu'elle met en lumière un parallèle entre l'évolution de la forme dramatique et celle de l'écriture de l'histoire dans les années 1840 , une étude de ce type nuance la théorie des scénarios-types de mise en intrigue de l'histoire développée par Hayden White dans Metahistory (1976). En effet, on voit ici la mise en intrigue varier chez un même auteur en fonction du moment où il écrit et des enjeux idéologiques et politiques de la sémantisation de l'histoire.

\section{NOTES}

1. Augustin Thierry, Récits des temps mérovingiens, Les Presses d'aujourd'hui, 1981, "Troisième récit », p. 131. Toutes les citations de cette œuvre renverront désormais à cette édition par le numéro de page entre parenthèses à la suite du texte.

2. Dans le livre qu'il consacre à l'historien, Augustin Thierry. Social and political consciousness in the evolution of a historical method (Paris, Droz, 1973), R. N. Smithson comparant les différentes éditions de la Conquête de l'Angleterre est sensible à l'évolution du style de Thierry à partir de la troisième édition, en 1830. « The imprudent, unrestrained and colorful style of 1825 has given way in numerous instances - but not in all - to a measured, balanced and cautious style. Many a fierce metaphor not caught in the 1826 revision has been replaced by the circumlocution of an « ardeur conquérante » or an " ardeur belliqueuse »; or by the not-always-unambiguous clarity of a similar classical periphrase. Undeniably the near-romantic stylist has evolved toward the classicist. » (p. 163). Smithson rapporte cette évolution au désir de Thierry de se faire élire à l'Académie des Inscriptions et Belles Lettres (et à son infirmité qui l'oblige à se soucier de sa position sociale).

3. Offrande de pain bénit à la fin de la messe. 
4. Ce qui, à l'échelle de chaque récit correspond au choix de l'ensemble de l'œuvre de s'organiser en récits détachés, en « masses de narration isolée » («Introduction », p.

27).

5. Grégoire de Tours, L'Histoire des rois francs, traduit du latin par J.J.E. Roy, Gallimard, «L'aube des peuples », 1968, p. 81.

6. Augustin Thierry, Histoire de la conquête de l'Angleterre par les Normands, $2^{\mathrm{e}}$ éd. revue et corrigée, Sautelet et cie, 1826.

7. VALERE

Donc tu prétends qu'ici règne la multitude?

BRUTE

Non, non ; ce nous serait une autre servitude

Le peuple turbulent, qui suit sa passion,

Est une proie acquise à chaque faction.

Celui qui sait le mieux flatter l'aveugle masse,

Entraîne son suffrage, et gouverne à sa place ;

Et les ambitions, mises en mouvement,

Ne produisent que trouble et que déchirement.

Laissons les sénateurs exercer leur tutelle ;

À nos patriciens laissons leur clientèle.

Il convient d'élever, par-dessus tous les fronts,

Des hommes que leur rang désigne pour patrons,

Afin qu'en de tels choix le bas peuple consulte

Cet indice éclatant plutôt que le tumulte.

(Lucrèce, Acte II, scène II)

\section{RÉSUMÉS}

Les Récits des temps mérovingiens d'Augustin Thierry ont remporté un grand succès dès leur parution en 1840 et pendant quelques décennies. Mais si l'on observe les particularités de ce texte, on s'aperçoit que le modèle de l'écriture de l'histoire a changé depuis l'entrée brillante de Thierry dans la carrière sous la Restauration avec ses Lettres sur l'Histoire de France et sa Conquête de l'Angleterre par les Normands. L'étude du rapport entre diégèse et narration, la mise en abyme du narrateur par la figure de Grégoire de Tours, l'effacement du modèle du drame et l'apparition corrélative d'un référence à la tragédie permettent de cerner l'évolution de la mise en intrigue de l'histoire et de formuler quelques hypothèses sur ce qui explique cette transformation.

\section{INDEX}

Mots-clés : historiographie, Augustin Thierry, Mérovingiens, drame, tragédie

Index chronologique : XIXe siècle 
AUTEUR

PAULE PETITIER

Université Paris Diderot - Paris 7 